\title{
Exploring the Influence of Vehicle Mobility on Information Spreading in VANETs
}

\author{
Zhigang $\mathrm{Li}^{1}$, Xin Wang ${ }^{1 *}$, Xinan Yue ${ }^{1}$, Yingli $\mathrm{Ji}^{2}$, and Hua Wang ${ }^{1}$ \\ ${ }^{1}$ School of Computer and Communication Engineering, Zhengzhou University of Light Industry, \\ Zhengzhou, 450002, China \\ [e-mail: Lizg.cn@hotmail.com, xinwang_m@163.com,1950572443@qq.com,2013016@zzuli.edu.cn] \\ ${ }^{2}$ Henan Co. Ltd, China Mobile Communications Group, Zhengzhou, 450008, China, \\ [e-mail: jiyingli@ha.chinamobile.com] \\ ${ }^{*}$ Corresponding author: Xin Wang
}

Received November 29, 2020; revised February 5, 2021; accepted February 16, 2021; published February 28, 2021

\begin{abstract}
With the advent of 5G communications, internet of vehicles technology has been widely used in vehicles. Then the dynamic spread of information between vehicles began to come into focus with more research. It is well known that the identification of nodes with great spread influence has always been a hot topic in the field of information spreading. Most of the existing work measures the propagation influence by degree centrality, betweenness centrality and closeness centrality. In this paper, we will identify influential vehicle nodes based on the mobility characteristics of vehicles to explore the information spreading between vehicles in VANETs. Different from the above methods, we mainly explore the influence of the radius of gyration and vehicle kilometers of travel on information spreading. We use a real vehicle trajectory data to simulate the information transmission process between vehicles based on the susceptible-infected-recovered SIR model. The experimental results show that the influence of information spreading does not enhance with increasing radius of gyration and vehicle kilometers of travel. The fact is that both the radius of gyration and the distance travelled have a significant influence on information spreading when they are close to the median. When the value of both is large or small, it has little influence on information spreading. In view of this results, we can use the radius of gyration and vehicle kilometers of travel to better facilitate the transmission of information between vehicles.
\end{abstract}

Keywords: Information Spreading, Vehicle Mobility, Radius of Gyration, Vehicle Kilometers of Travel, VANETs

This work was supported by the Science and technology project of henan province (Grant No. 192102210296) and the Science and technology project of henan province (Grant No. 202102210182) in China. 


\section{Introduction}

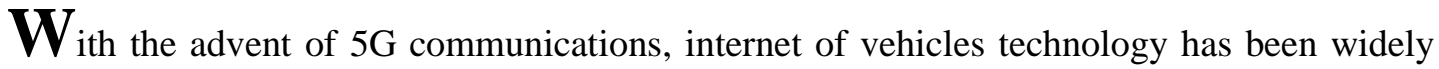
used in vehicles. Then the dynamic spread of information between vehicles began to come into focus with more research. In recent years, the identification of nodes with great spread influence has always been a hot topic in the field of information spreading. In complex networks, it is very important to determine the important nodes of information spreading, which can effectively control the spread of viruses in computer networks and diseases in social networks, as well as the spread of rumors, opinions, economic crisis and so on [1]. As we all know that there are many ways to communicate in Vehicular Ad Hoc Networks (VANETs) [2-4], for example between vehicles and vehicles (V2V), vehicles and infrastructure (V2I), vehicles and pedestrians (V2P), and vehicles and networks (V2N) [5]. In this paper, we focus on vehicle to vehicle transmission, and now we know that with the popularization and development of vehicle Internet technology, the information transmission between vehicles becomes more frequent. For example, vehicles can perceive multi-source perception information such as vehicles and road environment, and effectively extract and utilize dynamic information in the traffic environment to realize the sharing of perception information between vehicles [6]. Therefore, how to identify the nodes with greater influence of information transmission in VANETs has become a hot topic in the field of network information spreading.

With the continuous popularization of $5 \mathrm{G}$ network, the development of vehicle network technology. More and more information should be quickly and efficiently transmitted between vehicles, such as current road conditions, weather conditions and road traffic conditions, for real-time analysis to prevent traffic jams [7]. Most of the existing work measures the propagation influence by degree centrality, betweenness centrality, closeness centrality and other characteristics of network topology [8-12]. Some works, such as the greedy algorithm [13], the Node Rank algorithm [14], and the community-based algorithm [15], are conducted based on human mobility characteristics. Others are based on a topological diffusion model [16], molecular dynamics [17] or other methods. In this paper, we study the vehicle's radius of gyration [18] and vehicle kilometers of travel [19] to measure the spread influence of nodes. The radius of gyration and vehicle of travel are both vehicle mobility characteristics, so you can obtain more accurate measurements. We use a real larger vehicle trajectory data set to explore their influence on information transmission in VANETs.

To evaluate the influence of radius of gyration and vehicle kilometers of travel on information spreading, we use a real vehicle trajectory data to simulate the information transmission process between vehicles based on the susceptible-infected-recovered SIR [20] model. In this paper, our main contribution is the experimental results that we obtained. We found that the influence of information spreading does not enhance with increasing radius of gyration and vehicle kilometers of travel. But both have the greatest influence at a median value approximately between the maximum and minimum values, while radius of gyration and vehicle kilometers of travel has a relatively small spreading influence at larger and smaller values, and has certain regular volatility. Further, it is found that the degree of vehicles is also larger when the values of radius of gyration and vehicle kilometers of travel have greater spread influence, which is consistent with the characteristic that the range of information propagation is generally large when the node degree is large. Therefore, we can to better facilitate the spreading of information according to the range of radius of gyration and vehicle kilometers of travel of the vehicle.

This paper is organized as follows: Section 2 discusses related works. The mobility characteristics of vehicle radius of gyration and vehicle kilometers of travel are analyzed in 
section 3. In section 4, we present the experimental process of information spreading by mobility characteristics and discuss the experimental results. Finally, Section 5 concludes the paper.

\section{Related Works}

From previous research work, as we know, it has a great practical and theoretical significance to measure the spreaders spread ability. Most researchers identify influential spreaders based on the topology characteristics of the network. However, from the existing research work, the topology characteristics of networks such as the centrality measurement method have many limitations and shortcomings. D. Chen et al. [8] discovery only considered the local network structure information around the nodes in the degree centrality method, so the influential nodes could not be recognized occasionally. Although the betweenness centrality method takes global structural information into consideration, the main disadvantage of this method is that it is difficult to be applied in large-scale networks due to its large computational load [9]. When the network contains unrelated components, the distance between two nodes belonging to different components is not limited, which is the biggest problem with the closeness centrality method [10]. Since the eigen vector of the adjacency matrix is used to describe only one of its components, if there are two or more different components, using the eigenvector centrality method will result in an incorrect result. In addition, in the work [11], three centrality measures were redefined based on the time evolution graph model to identify influential nodes. In addition, Kitsak et al. [12] proved that k-core's influence on information dissemination is more accurate than usage degree or betweenness centrality. They found that the core node of the network has a large propagation influence, which is determined by its position in the network.

Moreover, according to the moving characteristics of vehicles, information spreading between vehicles is divided into different forms. At present, many researchers have studied the instantaneous information transmission in VANETs, and related research topics such as propagation speed, network connectivity, propagation distance and opportunity communication. Lili Du et al. [21] proposed an information-traffic-coupled cell transmission model () to capture information based on VANETs transmission dynamics. The IT-CTM method discretizes the road segment into multiple units and can track the dynamic movement of information, thus increasing its coverage. Moumena Chaqfeh et al. [22] propose and evaluate an effective multi-directional data distribution protocol (EDDP), which only relies on simple local data to indicate road conditions, so as to achieve better communication effect. In addition, mathematical methods are used to estimate the expectation, variance and probability distribution of information transmission distance on parallel roads [23-24]. It is assumed that vehicle headway follows poisson, gamma, or lognormal distribution. Clearly, this group of studies propagated the information on a snapshot of static traffic flow, because the information propagated by wireless communication was much faster than the traffic flow changes. Therefore, the influence of traffic dynamics on information dissemination has not been fully considered. Information is transmitted intermittently in VANETs. Since wireless communication is not available, the information may be exchanged by wireless communication or by the carrying vehicle. Wang et al. [25] put forward an analysis formula is proposed to estimate the information propagation time delay through the $\mathrm{V} 2 \mathrm{~V}$ communication network formed on one-way or two-way road sections with multiple lanes.

Furthermore, for the transmission of some emergency information in road traffic, such as ambulances, fire alarms, traffic accidents and other information. Some studies have explored 
the Urgent information spreading in VANETs. Yuanguo Bi et al. [26] propose an urban multihop broadcast protocol (UMBP) to spread emergency messages. In the first hop, two-way broadcast or multi-directional broadcast simultaneously performs the forwarding node selection scheme in different directions, and each direction successfully selects a single forwarding node. Directional broadcasting is then carried out at each hop in the direction of the message propagation until the emergency message reaches the intersection area, and then multi-directional broadcasting is carried out in the intersection area, finally making the emergency message seamlessly cover the target. Syed Sarmad Shah et al. [27] put forward the Time Barrier-Based Emergency Message Dissemination in Vehicular Ad-hoc Networks to reduce the message overhead that may disrupt the network. Propagate news in time through super-nodes. In order to avoid unnecessary broadcast problems caused by broadcast storms, time barrier technology is adopted to deal with this problem. Only the farthest vehicle relay can cover more distance information. Therefore, the message can reach the furthest node in a shorter time, thereby increasing coverage and reducing delay. In order to improve the transmission of emergency messages between vehicles, Marques et al. [28] put forward the strategy of using the location, direction, speed, the number of vehicle neighbors and the characteristics of urban areas, so that messages can reach all vehicles in the shortest time and at the lowest speed.

There are different kinds of information transmitted between vehicles in VANETs, and many scholars have proposed information transmission protocols between vehicles [29-31]. Korkmaz et al. [32] proposed a new and efficient multi-hop broadcast protocol (UMB) based on IEEE 802.11, aiming at solving the problems of the broadcast storm, hidden nodes and reliability of multi-hop broadcast in cities. In this protocol, the road part in the transmission range is divided into several segments, and the vehicles in the farthest non-empty segment without prior topology information are selected, so that the responsibility of forwarding and confirming broadcast data packets are assigned to only one vehicle. When there is an intersection in the path of message propagation, the vehicles at the intersection will launch a new directional broadcast. Dedicated Short Range Communication (DSRC) is a wireless communication technology designed for direct V2V connections, which takes the IEEE 802.11p standard as its physical (PHY) and medium access control (MAC) layers currently. DSRC can provide high-speed, real-time, accurate and reliable connections between vehicles [33]. Rakesh Shrestha et al. [34] proposed a new blockchain to solve the key message dissemination problem in VANET. We created a local blockchain for real event message exchange among vehicles in a country/region, which is a new blockchain suitable for VANET. We propose a common blockchain, which stores node credibility and message credibility in a distributed ledger suitable for secure message distribution.

Most of the above studies have used various methods to identify critical nodes in complex networks. For example, the characteristics of the network topology are studied to find the nodes that have an influence on information propagation. In VANETs, many researchers analyze the characteristics of vehicle motion to study the propagation of information between vehicles. Due to the limited transmission range and high mobility of vehicles, this will lead to rapid topology changes, resulting in partitioning and routing link failures. They study the information propagation in VANETs by analyzing transient, intermittent, and emergency information between vehicles. Many other scholars study information propagation between vehicles by analyzing the routing protocols in VANETs. As far as we know from existing works, no study has investigated information spreading in VANETs by analyzing the vehicle radius of gyration and vehicle kilometer traveled. Therefore, in the rest section, we will explore the effects of turn radius and vehicle kilometers on information propagation in VANETs. 


\section{Vehicle Mobility Characteristic Analysis}

In the research work of [18], M. C. Gonzalez et al. used the trajectory data of 100,000 anonymous mobile phone users for 6 months. In their research, they propose the concept of human mobility radius of gyration and analyzed its distribution. The experimental results show that the radius of gyration of user mobility approximates the truncated power law distribution. The network topology of VANETs changes rapidly due to the rapid movement of vehicles. Based on their research we first analyze the characteristics of radius of gyration of vehicle mobility used a real data set. In the meantime, we analyzed the characteristics of vehicle kilometers of travel and their influence on information spreading in VANETs.

In this paper, we used the dataset of mobility traces of taxi cabs in Rome, Italy [35]. Contributed by Lorenzo Bracciale, Marco Bonola, Pierpaolo Loreti, Giuseppe Bianchi, Raul Amici, Antonello Rabuffi. This dataset contains GPS coordinates of approximately 320 taxis collected over 30 days. We randomly selected 10 days of data to calculate radius of gyration and vehicle kilometers of travel for all vehicles and analyzed their distributions.

\subsection{Radius of Gyration Analysis}

In the supplementary data of [18], the calculation process of the radius of gyration is given. which is defined as follows:

$$
r_{g}^{a}(t)=\sqrt{\frac{1}{n_{c}^{a}(t)} \sum_{i=1}^{n_{c}^{a}}\left(r_{i}^{a}-r_{c m}^{a}\right)^{2}}
$$

Where $\boldsymbol{r}_{i}^{a}$ represents the $i=1, \ldots, n_{c}^{a}(t)$ positions recorded vector for vehicle $a$ and $\boldsymbol{r}_{c m}^{a}=1 / \boldsymbol{n}_{c}^{a}(\mathrm{t}) \sum_{i=1}^{n_{c}^{a}} \boldsymbol{r}_{i}^{a}(t)$ is the center vector of mass of the trajectory. This means that the user's movement in a period of time is around an axis of rotation, the radius of gyration is the vertical distance from the point of concentration to the axis of rotation.

We used 10 days of data to calculate the radius of gyration of all vehicles for three different time periods. The three time periods are 6 hours (15:00-21:00), 12 hours (8:00-20:00) and 24 hours (00:00-24:00) respectively. The average number of vehicles in three times is 148, 270 and 292 respectively. We calculated the average value of 10 times in each time period as the value of the radius of gyration. The distribution of the number of vehicles in different range of radius of gyration is shown in Table 1.

Table 1. The distribution of vehicles in different range of radius of gyration

\begin{tabular}{|c|c|c|c|c|c|c|}
\hline \multirow{2}{*}{$\begin{array}{c}\text { The distribution of } \\
\text { node }\end{array}$} & \multicolumn{7}{|c|}{ Radius of Gyration $(\mathbf{k m})$} \\
\cline { 2 - 7 } & $\mathbf{0 - 2}$ & $\mathbf{2 - 4}$ & $\mathbf{4 - 6}$ & $\mathbf{6 - 8}$ & $\mathbf{8 - 1 0}$ & $\mathbf{1 0 - 1 2}$ \\
\hline \hline 6 Hours & 21 & 44 & 55 & 11 & 14 & 3 \\
\hline 12 Hours & 39 & 74 & 116 & 20 & 19 & 2 \\
\hline 24 Hours & 40 & 66 & 138 & 23 & 22 & 3 \\
\hline
\end{tabular}

From Table 1, it can be found that most vehicles have a radius of gyration within 4-6km. A small number of vehicles have a smaller radius of gyration. Occasionally there are a few 
vehicles with large radius of gyration. Based on the distribution of vehicle rotation radius, which indicating that most of the users do the reciprocal motion in the intermediate area, and only a small number of users are active in the larger and smaller areas.

\subsection{Vehicle Kilometers of Travel Analysis}

The vehicle kilometers of travel refer to the total kilometers traveled by vehicles on a roadway. In traffic and transport planning the vehicle kilometers of travel is the most frequently used characteristic to consider for various purposes such as estimating vehicle emissions, resource allocation, analyzing crashes, assessing traffic impact, making road safety policies, and road allocating resources. We analyzed the vehicle mobility characteristics by calculating vehicle kilometers of travel, which is defined as follows :

$$
\boldsymbol{S}^{a}=\sum_{i=1}^{n_{t}^{a}}\left(\boldsymbol{r}_{i+1}^{a}-\boldsymbol{r}_{i}^{a}\right)
$$

Where $\boldsymbol{r}_{i}^{a}$ represents the $i=1, \ldots, n_{c}^{a}(t)$ positions recorded vector for vehicle $a$. We still select 10 days of data from the dataset. We use (2) to calculate the average vehicle kilometers of travel of each vehicle for three different time periods of 6 hours (15:00-21:00), 12 hours (8:00-20:00), and 24 hours (00:00-24:00) in a 10-day period. The average number of vehicles in three times is 148,270 and 292 respectively. The distribution of the number of vehicles in different range of vehicle kilometers of travel, as shown in Table 2.

Table 2. The distribution of vehicles in different range of vehicle kilometers of travel

\begin{tabular}{|c|c|c|c|c|c|}
\hline \multirow{5}{*}{$\begin{array}{r}\text { The distribution of } \\
\text { nodes }\end{array}$} & \multicolumn{5}{|c|}{ Vehicle Kilometers of Travel (km) } \\
\cline { 2 - 6 } & $\mathbf{0 - 5 0}$ & $\mathbf{5 0 - 1 0 0}$ & $\mathbf{1 0 0 - 1 5 0}$ & $\mathbf{1 5 0 - 2 0 0}$ & $\mathbf{2 0 0 - 2 5 0}$ \\
\hline \hline 6 Hours & 35 & 74 & 28 & 9 & 2 \\
\hline 12 Hours & 45 & 136 & 66 & 20 & 3 \\
\hline 24 Hours & 20 & 97 & 94 & 58 & 23 \\
\hline
\end{tabular}

From Table 2, it can be seen that the distribution of vehicle kilometers of trave is concentrated in $50-100 \mathrm{~km}$. Only a small number of vehicles have more and less vehicle kilometers of trave. This indicates that most vehicles have a relatively normal vehicle kilometers of trave, only a small number of vehicles have less and more travel distance.

\section{Information Spreading Experiments and Results Analysis}

In our experiment, we from several classical disease spreading models select the susceptibleinfected-recovered (SIR) model to study the information spreading process. The SIR model is applicable to people who have Susceptible, Infected, and Recovered and who are cured of a disease that does not recur. Susceptible and Infected effectively come into contact with each other and become Infected, which can be cured and become Recovered, with no latency period and lifelong immunity. The reason why we choose the SIR model is to avoid double-counting the infected nodes. In other words, the nodes whose information has been spread do not accept spreading, so as to avoid double-counting the nodes that have been spread. One day is the 
smallest time unit in the model. The total number of people is $\mathrm{N}$. The total number of people is constant, irrespective of births and deaths, in-migration, and out-migration. The ratio of each type of population to the total number of people at time $t$ is given as $\mathrm{s}(t), i(t)$, and $r(t)$, and the number of each type of population is given as $S(t), I(t)$, and $R(t)$ respectively. At the initial moment $t=0$, the initial ratios are $\mathrm{s}, i_{0}$, and $r_{0}$. daily exposure $\lambda$, which is the average number of susceptible people each patient is effectively exposed to each day. The daily cure rate $\mu$ is the ratio of the number of patients cured per day to the total number of patients. Average cure days $1 / \mu$, also known as the average duration of infection, is the number of days from the disease to cure. The number of people exposed during the infection period $\sigma=\lambda / \mu$, that is, the number of susceptible people effectively exposed to each patient within $1 / \mu$ of the entire infection period.

In VANETs, the process of information spreading between vehicles, where the spreader is $\mathrm{S}$ and the receiver is I, is similar to infectious disease for the same message, and in the SIR model, the infected person is cured and no longer infected with the virus. Therefore, for the same message, the receiver will no longer receive it after receiving it, and it is called R. First of all, we notice that all nodes with the exception of the infectious nodes are initially susceptible. In each step, each infected node tries to recover after infecting its sensitive neighbor with probability $\beta$ of infection, with probability $\gamma$ of recovery. In this state, on average, one infected node will contact $k$ neighbors before recovery, so the probability of recovery of infected nodes is $\gamma=1 / k$. The recovered node will never receive a message from another infected node. Here, the selection of the SIR model can avoid the redundancy of the copies of information generated during the information transmission process. In this paper, the probability of susceptible individuals is assumed to be $\beta=1, s(t), i(t)$ and $r(t)$ are the proportion of susceptible nodes, infective nodes and recovery nodes in the whole node respectively. The coupled differential equations of SIR model is as follow:

$$
\left\{\begin{array}{l}
d i / d t=\beta s i-\gamma i, i(0)=1 / \mathrm{n} \\
d s / d t=-\beta s i, s(0)=1-1 / \mathrm{n} \\
d r / d t=\gamma i, \mathrm{r}(0)=0
\end{array}\right.
$$

When $d r / d t \neq 0, r(t)$ does not reach a steady-state. At this time, the proportion of spreading $(\mathrm{PoS})$ is equal to the following formula:

$$
P o S=i(t)+r(t)=1-s(t)
$$

\subsection{Results Analysis of Radius of Gyration on Information Spreading}

In this experiment, we are divided into 12 intervals according to the value of the radius of gyration. Each experiment randomly selects 1 vehicle from each interval as the proportion node, and repeat the selection 5 times. If the distance between a vehicle and the proportion node is less than $400 \mathrm{~m}$ in 12 seconds before and after, we shall deem that the information is spreading to the vehicle. We use the SIR model to calculate the average propagation rate of 5 
nodes randomly selected in each interval.

As you can see in Fig. 1, we use red, blue and green to represent the different radius of gyration calculation periods. From the figure, we can see that the larger spread period has a larger standard deviation. Here, the deadline for information spreading is the same as the radius of gyration period. Therefore, there is an obvious conclusion that the proportion of spreading over the 24 hours radius of gyration time span is significantly larger than the radius of gyration of 12 hours or 6 hours, radius of gyration is almost consistent with the fluctuation of regularity. In general, in the initial stage, the proportion of spreading tends to increase with the increase of the radius of gyration, and the proportion of spreading reaches its peak when the radius of gyration of three time periods is nearly $6 \mathrm{~km}$. Then the proportion of information spread gradually decreases.

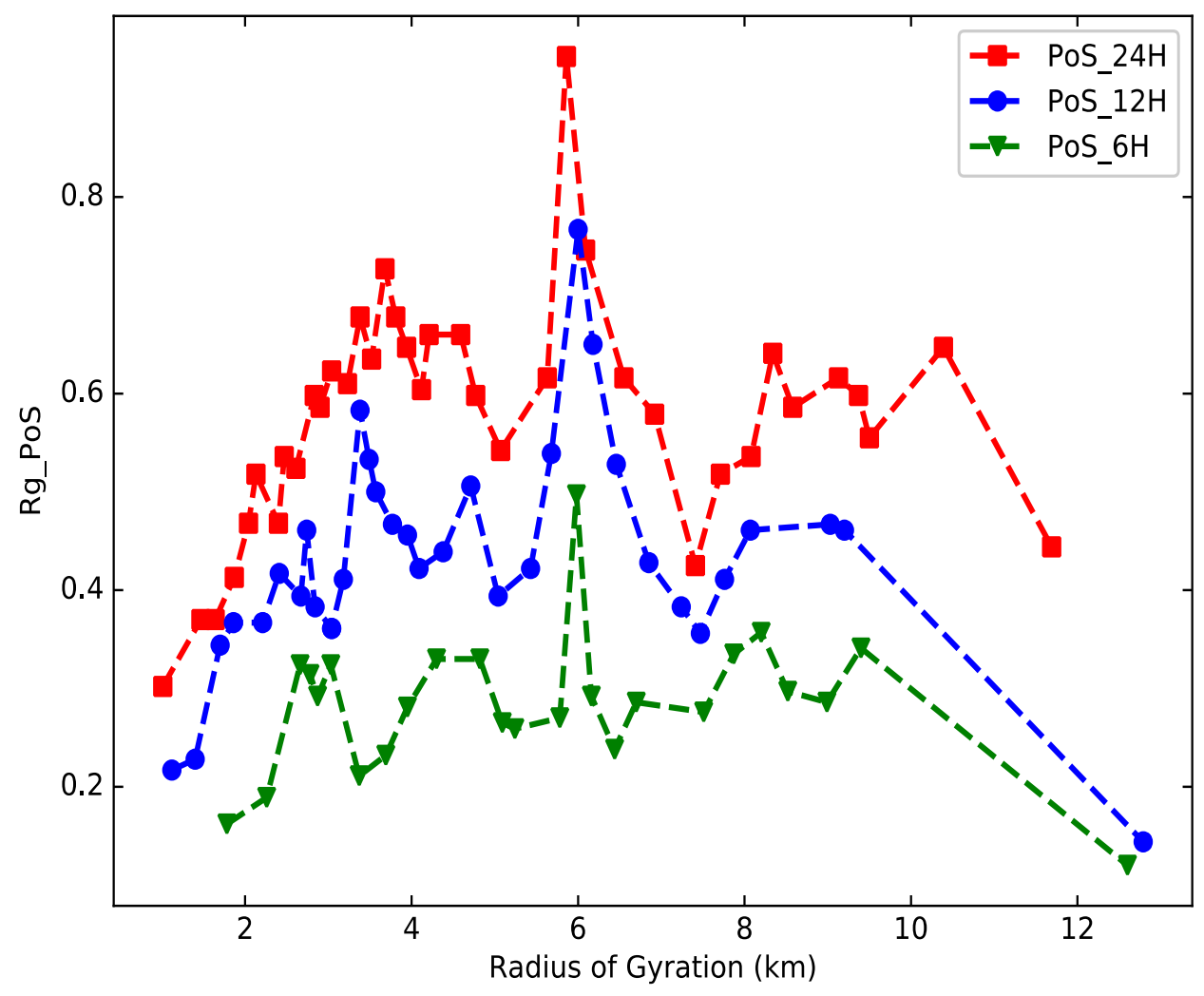

Fig. 1. The PoS of radius of gyration in different periods

The PoS of the radius of gyration in the three time periods showed a trend of first increasing and then decreasing. From this we can conclude that the influence of information spreading does not enhance with increasing radius of gyration. The fact is that the radius of gyration have a significant influence on information spreading when it is close to the median. When the value of both is large or small, it has little influence on information spreading. The single transverse region is limited when the radius of gyration is small, so the spreading is relatively small. However, we speculate that the individual is in the periphery of the area when the radius of gyration is large, which is not the area with high user activity. 
We not only analyzed the information PoS of the radius of gyration, but also studied the influence of the radius of gyration on the spreading synchronously from the geographical range. Nodes with large and small influence of radius of gyration on spreading were selected as infection nodes. For the convenience of examples, we only show the spreading range of the radius of gyration of four nodes, which are about $3 \mathrm{~km}, 5.9 \mathrm{~km}, 6.6 \mathrm{~km}$ and $8.3 \mathrm{~km}$ on the 24 hours period respectively. In Fig. 2, we represent infected and uninfected nodes in red and blue dots, respectively. It can be seen from the figure that the number of infected nodes reaches the maximum when the radius of gyration is about $5.9 \mathrm{~km}$. When the radius of gyration is approximately $6.6 \mathrm{~km}$, the spread range is larger, which is less than the radius of gyration is about $5.9 \mathrm{~km}$. However, when the radius of gyration is about $3 \mathrm{~km}$ and $8.3 \mathrm{~km}$, the number of infected nodes is relatively small.
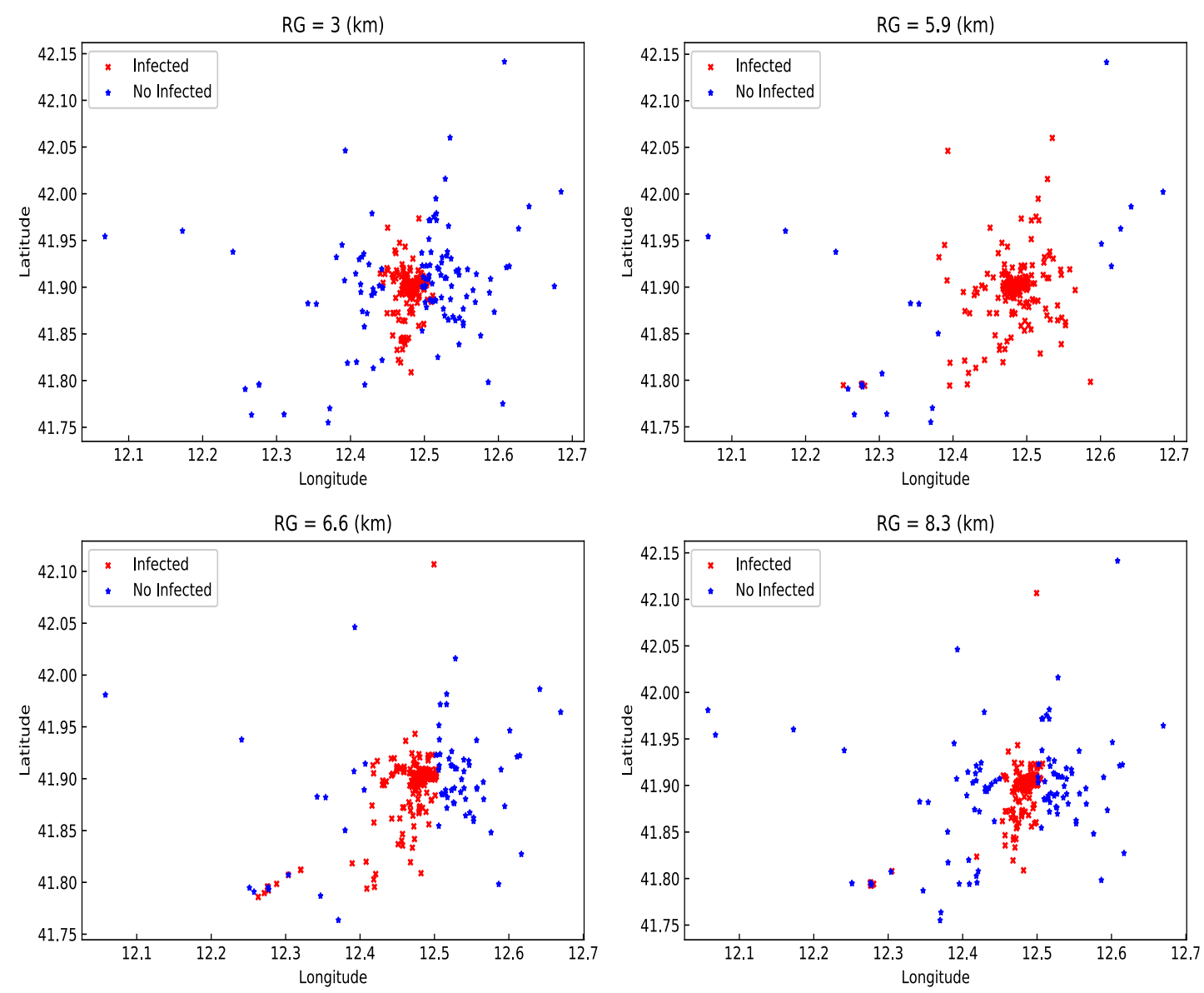

Fig. 2. The information spreading range of different radius of gyration values

\subsection{Results Analysis of Vehicle Kilometers of Travel on Information Spreading}

We discussed the effects of vehicle kilometers of travel on information spreading. As you can see in Fig. 3, we use red, blue and green to represent the different vehicle kilometers of travel calculation periods. As you can see from the graph, larger periods have larger standard deviations. We use the same deadline for information spreading as the vehicle kilometers of travel. There is a conclusion similar to the radius of gyration. The proportion of information 
spreading over the 24 hours vehicle kilometers of travel time span is significantly larger than vehicle kilometers of travel of 12 hours or 6 hours, vehicle kilometers of travel is almost consistent with the fluctuation of regularity. In general, the vehicle kilometers of travel have an increasing trend, and the fluctuation amplitude of vehicle kilometers of travel on information spreading is consistent with radius of gyration. The proportion of information spread over three time periods peaked when the vehicle kilometers of travel about $88 \mathrm{~km}$.Since then, the proportion of information spreading has declined. We also studied the influence of vehicle kilometers of travel on propagation geographically.

The influence of vehicle kilometers of travel is also analyzed on transmission in terms of the proportion of information transmitted, we also studied the geographic extent of transmission of vehicle kilometers of travel. We selected nodes where vehicle kilometers of travel had a greater or lesser propagation impact as infection nodes. We counted four nodes whose vehicle kilometers traveled in a 24-hour period were approximately 30, 88, 91, and 207 kilometers, respectively. In Fig. 4, in the same way that we represent infected and uninfected nodes in red and blue dots, respectively. It can be seen from the figure that the number of infected nodes reaches the maximum when the vehicle kilometers of travel is about $88 \mathrm{~km}$. When vehicle kilometers of travel is about $91 \mathrm{~km}$, the spread is larger but still smaller than when vehicle kilometers of travel is about $88 \mathrm{~km}$. In contrast, when vehicle kilometers of travel is about $30 \mathrm{~km}$ or $207 \mathrm{~km}$, only a few nodes are infected.

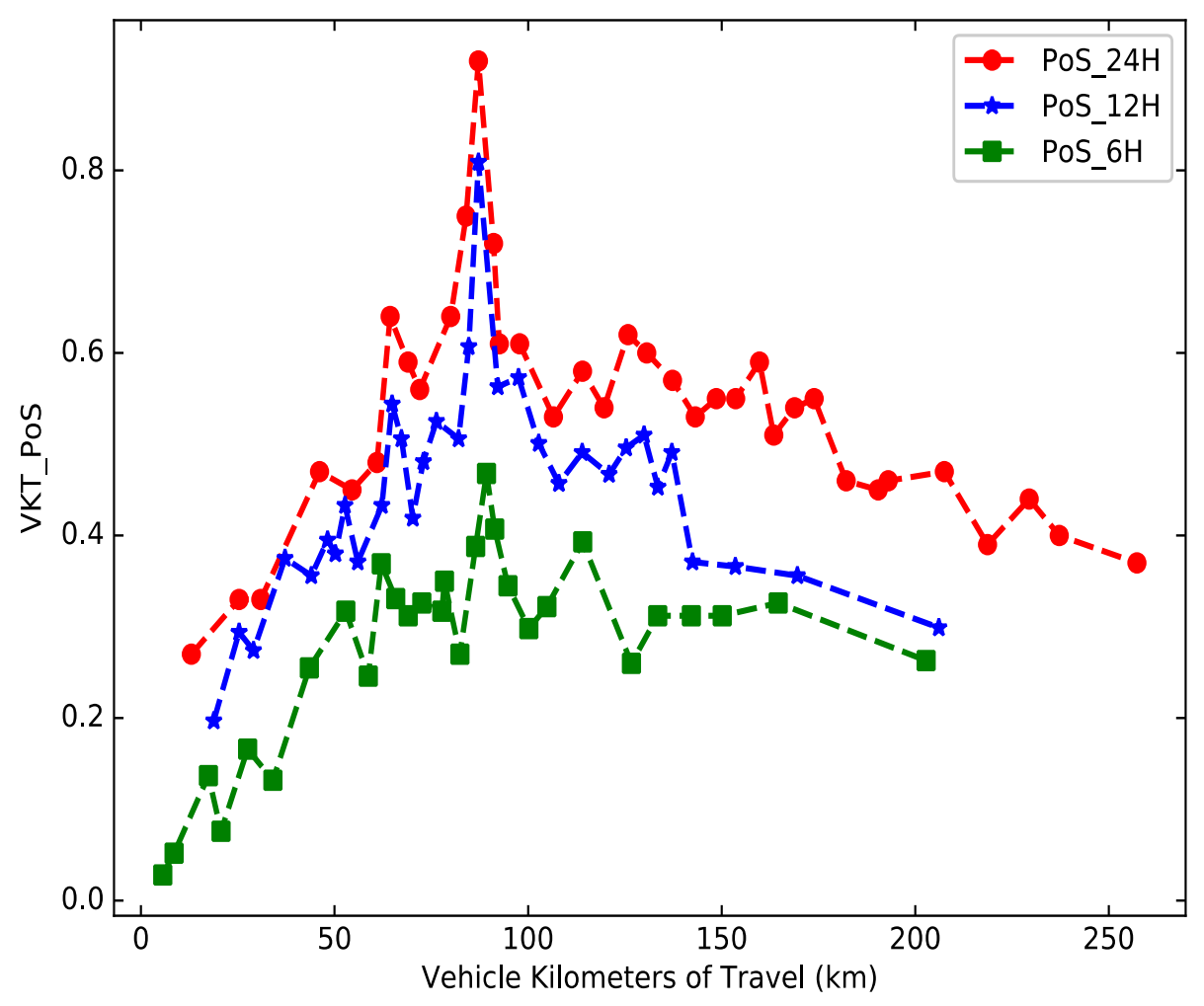

Fig. 3. The PoS of vehicle kilometers of travel in different periods 


\section{Conclusion}

With the development of 5G technology, the application of 5G in VANET improves the communication mode and communication quality of vehicle network. Information spreading in dynamic ad hoc networks has become a hot topic. In this paper, according to the moving characteristics of vehicles, the radius of gyration and kilometers traveled by vehicles are selected to explore the influence of vehicle movement on information dissemination in VANETs. We use a real vehicle trajectory data to simulate the information transmission process between vehicles based on the susceptible-infected-recovered SIR model. The influence of information spreading will not increase with the increase of vehicle radius of gyration and kilometers traveled. The fact is that both the radius of gyration and the distance travelled have a significant influence on information spreading when they are close to the median. When the value of both is large or small, it has little influence on information spreading. In view of this results, we can use the radius of gyration and vehicle kilometers of travel to better facilitate the transmission of information between vehicles. In the following work, we expect to study more about the influence of vehicle mobility characteristics on information spreading.
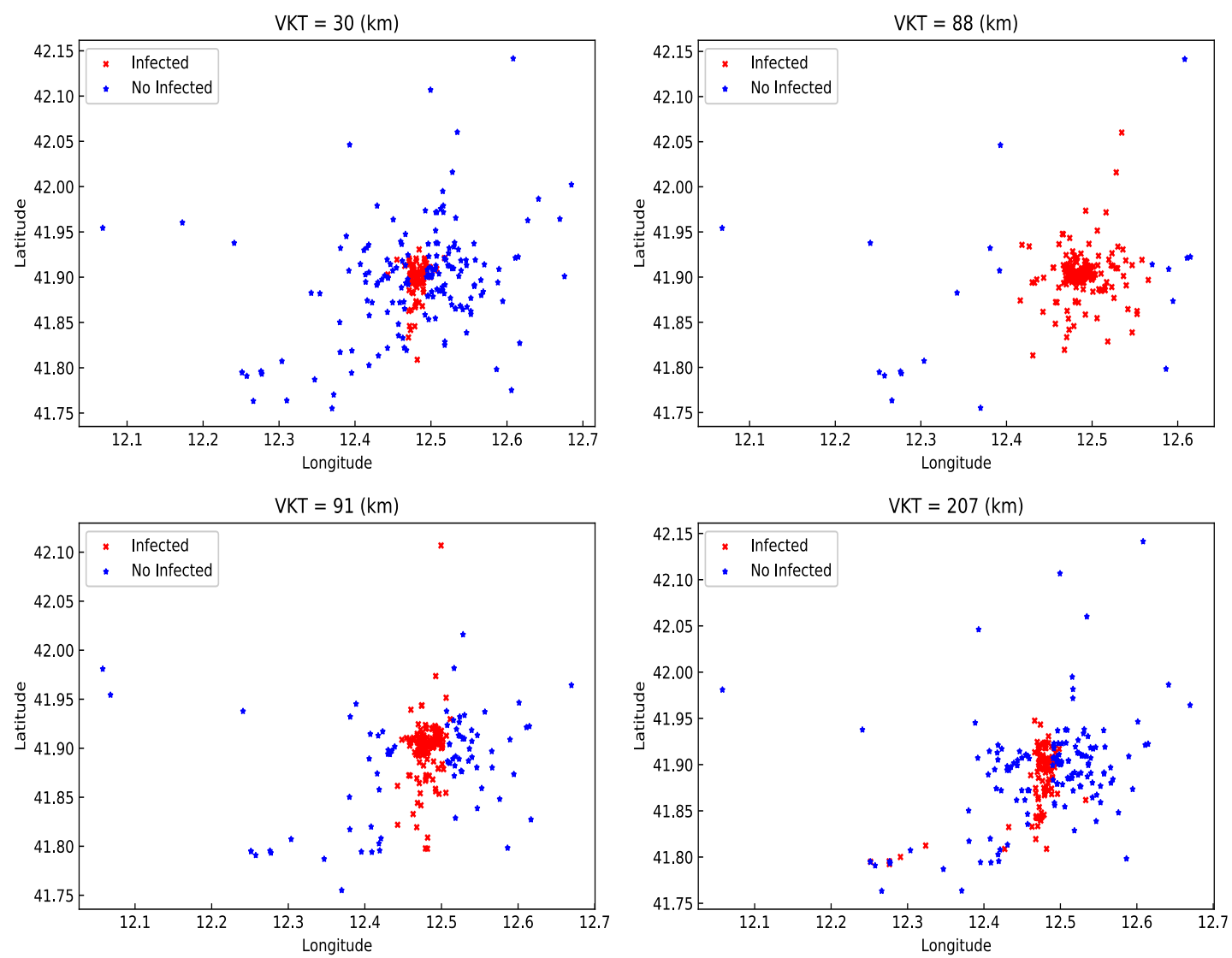

Fig. 4. The information spreading range of different vehicle kilometers of travel values 


\section{References}

[1] W. Song, S. U. Rehman, and M. B. Awan, "Road Aware Information Sharing in VANETs," KSII Transactions on Internet \& Information Systems, vol. 9, no. 9, pp. 3377-3395, Sep. 2015. Article (CrossRef Link)

[2] X. L. Wang, J. J. Jiang, S. Zhao, and X. Bai, "A Fair Blind Signature Scheme to Revoke Malicious Vehicles in VANETs,” Computers, Materials \& Continua, vol. 58, no. 1, pp. 249-262, Jan. 2019. Article (CrossRef Link)

[3] D. Y. Kim and S. Kim, "A Data Download Method from RSUs Using Fog Computing in Connected Vehicles," Computers, Materials \& Continua, vol. 59, no. 2, pp. 375-387, Feb. 2019. Article (CrossRef Link)

[4] B. Mohammed and D. Naouel, "An Efficient Greedy Traffic Aware Routing Scheme for Internet of Vehicles," Computers, Materials \& Continua, vol. 60, no. 3, pp. 959-972, Sep. 2019. Article (CrossRef Link)

[5] L. Lü, D. Chen, X. L. Ren, Q. M. Zhang, Y. C. Zhang, and T. Zhou, "Vital nodes identification in complex networks," Physics Reports-review Section of Physics Letters, vol. 650, pp. 1-63, Sep. 2016. Article (CrossRef Link)

[6] S. Al-Sultan, M. M. Al-Doori, A. H. Al-Bayatti, and H. Zedan, "A comprehensive survey on vehicular ad hoc network," Journal of network and computer applications, vol. 37, pp. 380-392, Jan. 2014. Article (CrossRef Link)

[7] J. Shen, T. Zhou, J. Lai, P. Li, and S. Moh, "Secure and Efficient Data Sharing in Dynamic Vehicular Networks," IEEE Internet of Things Journal, vol. 7, no. 9, pp. 8208-8217, Sep. 2020. Article (CrossRef Link)

[8] D. Chen, L. Lü, M. S. Shang, Y. C. Zhang, and T. Zhou, "Identifying influential nodes in complex networks," Physica a: Statistical Mechanics and its Applications, vol. 391, no. 4, pp. 1777-1787, Feb. 2012. Article (CrossRef Link)

[9] D. Wei, X. Deng, X. Zhang, Y. Deng, and S. Mahadevan, "Identifying influential nodes in weighted networks based on evidence theory," Physica A: Statistical Mechanics and its Applications, vol. 392, no. 10, pp. 2564-2575, May 2013. Article (CrossRef Link)

[10] Y. Du, C. Gao, Y. Hu, S. Mahadevan, and Y. Deng, "A new method of identifying influential nodes in complex networks based on TOPSIS," Physica A: Statistical Mechanics and its Applications, vol. 399, pp. 57-69, Apr. 2014. Article (CrossRef Link)

[11] Z. Gao, Y. Shi, and S. Chen, "Measures of node centrality in mobile social networks," International Journal of Modern Physics C, vol. 26, no. 09, Sep. 2015. Article (CrossRef Link)

[12] M. Kitsak, L. K. Gallos, S. Havlin, F. Liljeros, L. Muchnik, H. E. Stanly, and H. A. Makse, "Identification of influential spreaders in complex networks," Nature physics, vol. 6, no. 11, pp. 888-893, Nov. 2010. Article (CrossRef Link)

[13] B. Han, P. Hui, V. S. Kumar, and J. Zhao, "Cellular traffic offloading through opportunistic communications: a case study," in Proc. of the $5^{\text {th }}$ ACM Workshop on Challenged Networks, pp. 31-38, Sep. 2010. Article (CrossRef Link)

[14] Z. Li, Y. Shi, S. Chen, and J. Zhao, "Cellular Traffic Offloading through Opportunistic Communications Based on Human Mobility," KSII Transactions on Internet \& Information Systems, vol. 9, no. 3, pp. 872-885, Mar. 2015. Article (CrossRef Link)

[15] Y. J. Chuang and C. J. Lin, "Cellular traffic offloading through community-based opportunistic dissemination," in Proc. of IEEE Wireless Communications and Networking Conference, pp. 3188-3193, Apr. 2012. Article (CrossRef Link)

[16] D. Kempe, J. Kleinberg, and É. Tardos, "Maximizing the spread of influence through a social network," in Proc. of the $9^{\text {th }}$ ACM SIGKDD International Conference on Knowledge Discovery and Data Mining, pp. 137-146, Aug. 2003. Article (CrossRef Link)

[17] M. C. González and H. J. Herrmann, "Scaling of the propagation of epidemics in a system of mobile agents," Physica A: Statistical Mechanics and its Applications, vol. 340, no. 4, pp. 741748, Sep. 2004. Article (CrossRef Link) 
[18] M. C. Gonzalez, C. A. Hidalgo, and A. L. Barabasi, "Understanding individual human mobility patterns," Nature, vol. 453, no. 7196, pp. 779-782, June 2008. Article (CrossRef Link)

[19] Y. Zhao and K. M. Kockelman, "Anticipating the regional impacts of connected and automated vehicle travel in Austin, Texas," Journal of Urban Planning and Development, vol. 144, no. 4, Dec. 2018. Article (CrossRef Link)

[20] C. Liu and Z. K. Zhang, "Information spreading on dynamic social networks," Communications in Nonlinear Science and Numerical Simulation, vol. 19, no. 4, pp. 896-904, Apr. 2014. Article (CrossRef Link)

[21] L. Du, S. Gong, L. Wang, and X. Y. Li, "Information-traffic coupled cell transmission model for information spreading dynamics over vehicular ad hoc network on road segments," Transportation Research Part C: Emerging Technologies, vol. 73, pp. 30-48, Dec. 2016. Article (CrossRef Link)

[22] M. Chaqfeh, H. El-Sayed, and A. Lakas, "Efficient data dissemination for urban vehicular environments," IEEE Transactions on Intelligent Transportation Systems, vol. 20, no. 4, pp. 12261236, Aug. 2018. Article (CrossRef Link)

[23] B. X. Wang, K. Yin, and Y. Zhang, "An exact Markov process for multihop connectivity via intervehicle communication on parallel roads," IEEE Transactions on Wireless Communications, vol. 11, no. 3, pp. 865-868, Mar. 2012. Article (CrossRef Link)

[24] K. Yin, X. B. Wang, and Y. Zhang, "Vehicle-to-vehicle connectivity on two parallel roadways with a general headway distribution," Transportation Research Part C: Emerging Technologies, vol. 29, pp. 84-96, Apr. 2013. Article (CrossRef Link)

[25] W. Wang, S. S. Liao, X. Li, and J. S. Ren, "The process of information propagation along a traffic stream through intervehicle communication," IEEE Transactions on Intelligent Transportation Systems, vol. 15, no. 1, pp. 345-354, Feb. 2013. Article (CrossRef Link)

[26] Y. Bi, H. Shan, X. S. Shen, N. Wang, and H. Zhao, "A multi-hop broadcast protocol for emergency message dissemination in urban vehicular ad hoc networks," IEEE Transactions on Intelligent Transportation Systems, vol. 17, no. 3, pp. 736-750, Mar. 2015. Article (CrossRef Link)

[27] S. S. Shah, A. W. Malik, and A. U. Rahman, "Time barrier-based emergency message dissemination in vehicular ad-hoc networks," IEEE Access, vol. 7, pp. 16494-16503, Jan. 2019. Article (CrossRef Link)

[28] M. Marques, C. Senna, and S. Sargento, "Evaluation of Strategies for Emergency Message Dissemination in VANETs," in Proc. of IEEE Symposium on Computers and Communications, pp. 1-6, July 2020. Article (CrossRef Link)

[29] Y. Zhang, Y. Deng, Y. Liu, and L. Wang, "Dynamics Modeling and Stability Analysis of Tilt Wing Unmanned Aerial Vehicle During Transition," Computers, Materials \& Continua, vol. 59, no. 3, pp.833-851, June 2019. Article (CrossRef Link)

[30] X. Duan, J. Wei, D. Tian, J. Zhou, H. Xia, X. Li, and K. Zheng, "Adaptive Handover Decision Inspired By Biological Mechanism in Vehicle Ad-hoc Networks," Computers, Materials \& Continua, vol. 61, no. 3, pp. 1117-1128, Dec. 2019. Article (CrossRef Link)

[31] Q. Wang, C. Yang, S. Wu, and Y. Wang, "Lever Arm Compensation of Autonomous Underwater Vehicle for Fast Transfer Alignment," Computers, Materials \& Continua, vol. 59, no. 1, pp. 105118, Apr. 2019. Article (CrossRef Link)

[32] G. Korkmaz, E. Ekici, F. Özgüner, and U. Ozguner, "Urban multi-hop broadcast protocol for intervehicle communication systems," in Proc. of the $1^{\text {st }}$ ACM International Workshop on Vehicular ad Hoc Networks, pp. 76-85, Oct. 2004. Article (CrossRef Link)

[33] M. A. Al-Absi, A. A. Al-Absi, and H. J. Lee, "Comparison between DSRC and other Short Range Wireless Communication Technologies," in Proc. of the $22^{\text {nd }}$ International Conference on Advanced Communication Technology, pp. 1-5, Feb. 2020. Article (CrossRef Link)

[34] R. Shrestha, R. Bajracharya, A. P. Shrestha, and S. Y. Nam, "A new type of blockchain for secure message exchange in VANET," Digital Communications and Networks, vol. 6, no. 2, pp. 177-186, May 2020. Article (CrossRef Link)

[35] L. Bracciale, M. Bonola, P. Loreti, G. Bianchi, R. Amici, and A. Rabuffi, "The roma/taxi dataset," A Community Resource for Archiving Wireless Data, July 2014. Article (CrossRef Link) 


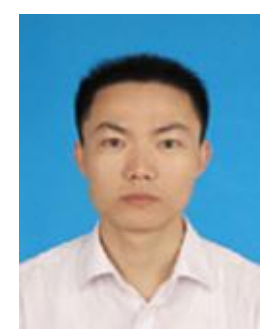

Zhigang Li received the Ph.D. degree from Beijing University of Posts and Telecommunications (BUPT) in 2016. He is currently a research staff of Zhengzhou University of Light Industry (ZZULI), Henan, China. His current research interests include mobile computing, cellular network, VANETs and C-V2X.

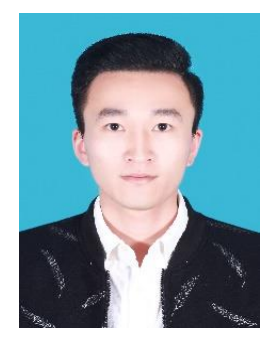

Xin Wang is currently working toward a master's degree with the School of Computer and Communication Engineering, Zhengzhou University of Light Industry (ZZULI), Henan, China. His current research interests include VANETs and C-V2X.

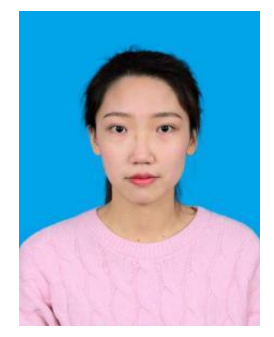

Xinan Yue is currently working toward a master's degree with the School of Computer and Communication Engineering, Zhengzhou University of Light Industry (ZZULI), Henan, China. Her current research interests include VANETs and C-V2X.

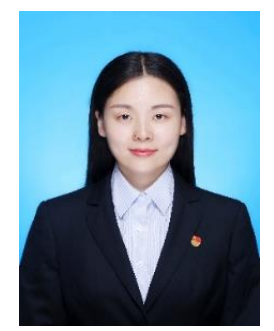

Yingli Ji received the M.S. degree from central China Normal University in 2013. She works in China Mobile Group Henan Company Limited(CMHA), Her current research interests include mobile computing, cellular network.

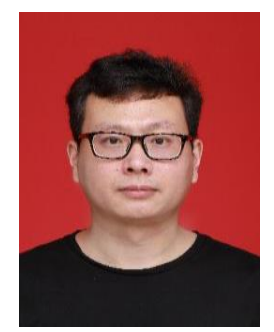

Hua Wang received the Ph.D. degree from Wuhan University in 2013. He is currently a research staff of Zhengzhou University of Light Industry (ZZULI), Henan, China. His current research interests include applications of artificial intelligence, spatial optimization decision, GIS. 